\title{
Effets d'Acacia senegal (L.) Willd. sur le Rendement du Niébé (Vigna unguiculata) au Niger, Afrique de l'ouest
}

\author{
Addam Kiari Saidou, \\ Moussa Hassane, \\ Institut National de la Recherche Agronomique du Niger, \\ INRAN, Niamey, Niger \\ Youchaou Tawaye Abdoullatif, \\ Ambouta Karimou,
}

Faculté d'Agronomie, Université Abdou Moumouni de Niamey, Niger

Doi: 10.19044/esj.2018.v14n27p176 URL:http://dx.doi.org/10.19044/esj.2018.v14n27p176

\begin{abstract}
This paper focuses on the influence of Acacia senegal on soil and cowpea production (Vigna unguiculata) at the N'Dounga Forest Station (INRAN) through a complete randomized block design. Treatments based on its association with cowpea in isolated trees and planting were applied. Chemical parameters, nitrogen fixation potential, and cowpea production were evaluated under these treatments. The results show an improvement in the chemical property of the soil in the presence of A. Senegal. Since the $\mathrm{pH}$ is acidic irrespective of the modality, the analysis of the variance shows a highly significant difference between the control and the other treatments for the water $\mathrm{pH}(\mathrm{P}<0.0001)$. Also, the nitrogen fixation potential cowpea was better expressed in his presence. However, the A. Senegal has less influenced the production of cowpea. The yields of pods, seeds, and fodder were reduced compared to the control. This study also revealed the dynamics of mineral elements after harvests, which reassures producers about the sustainability of the tree/crop system.
\end{abstract}

Keywords: Soil, fertility, Acacia senegal, cowpea, N'Dounga Station

\section{Résumé}

Une étude portant sur l'influence d' Acacia senegal sur le sol et la production de niébé (Vigna unguiculata) a été menée à la station forestière de N'Dounga (INRAN) à travers un dispositif en bloc complet randomisé. Des traitements portant sur son association avec le niébé en arbre isolé et en 
plantation ont été appliqués. Les paramètres chimiques, le potentiel de fixation d'azote et la production du niébé ont été évalués sous ces traitements. Les résultats qui en sont issus montrent une amélioration de la propriété chimique du sol en présence d'A. senegal. Vue que le $\mathrm{pH}$ est acide quelle que soit la modalité, et que l'analyse de la variance montre une différence hautement significative entre le control et les autres traitements pour le $\mathrm{pH}$ eau $(\mathrm{P}<0.0001)$, Le potentiel de fixation d'azote du niébé s'est mieux exprimé en sa présence. Cependant, l'A. senegal a moins influencé la production du niébé ; les rendements des gousses, graines et fanes étaient réduits par rapport au témoin. Cette étude a aussi permis de constater la dynamique des éléments minéraux après les récoltes, ce qui rassure les producteurs sur la durabilité du système arbre/culture.

Mots-clés: Sol, fertilité, Acacia senegal, niébé, Station N'Dounga

\section{Introduction}

L'agriculture nigérienne est une agriculture de subsistance avec peu d'apport de fumure organique et minérale (Ibrahim, 2010). La consommation d'engrais minéraux au Niger est extrêmement faible ; en moyenne, elle est de l'ordre de $20.000 \mathrm{t}$ sur 5,35 millions d'hectares soit 4,3 $\mathrm{kg} \mathrm{ha}^{-1}$ cultivé et par an (RECA, 2010). Cet état de fait trouve son explication dans l'inaccessibilité de cet intrant et la pauvreté de la population. Pourtant, même s'ils sont accessibles, les engrais minéraux seuls ne suffisent pas, sur le long terme, à maintenir la fertilité des sols ou augmenter le rendement des cultures (Bado, 2002). En effet, nombreuses études ont montré leurs effets négatifs à long terme sur la dégradation chimique des sols, entraînant de ce fait une réduction de rendement (Koulibaly et al., 2010). Face à cette situation, les systèmes de cultures plus durables à faibles intrants et les processus naturels doivent être exploités. A ce titre, les ligneux apparaissent comme une alternative importante à prendre en compte, notamment leur utilisation technologique à travers un recyclage approprié de la biomasse produite par l'arbre en association avec la plante cultivée (Zouboudré et al., 2005).

Les arbres protègent et améliorent les cultures agricoles et les conditions de vie dans les villages (Larwanou et al., 2010).

L'avantage de l'intégration de l'arbre dans les systèmes de cultures a été souligné par plusieurs auteurs :

Dans les zones sèches l'arbre influence la composition floristique de la strate herbacée ou cultivée. Cet impact positif est lié essentiellement à l'amélioration des conditions edapho-climatiques sous ombrage (Grousis \& Akpo, 2006). Certains arbres permettent le maintien de la fertilité des sols, et la durabilité des systèmes de cultures. En effet, la biomasse produite par ces arbres est décomposée, ce qui permet un recyclage des nutriments pompés par 
les arbres des horizons profonds vers les horizons de surface (Gbemavo et al., 2010). Certains ligneux, en plus de leur effet fertilisant, accroîtraient la teneur en eau pondérale et contribuent à la limitation de la transpiration des cultures sous-jacentes et l'évaporation du sol (Zomboudré et al., 2005).

L'impact de A. senegal sur les changements des propriétés du sol a été largement documenté (El Tahir et al., 2009; Samb, 2010). En association avec les cultures, l'A. senegal ne présente pas de compétition. Il produit des racines pivotantes qui peuvent exploiter l'eau et les nutriments jusqu'à 30m de profondeur, mais présente par contre une concurrence pour la lumière (Boubacar, 2006). S'agissant de la productivité des cultures associées à A.senegal, les effets de cet arbre semblent être controversés. Ainsi, les résultats obtenus sont tantôt meilleurs, au fur et à mesure que l'on s'éloigne du tronc, tantôt neutres (Raddad \& luukannen, 2006) et mauvais (El Tahir et al., 2009). Cependant, selon PACII (2011), les paysans évitent de semer sous le houppier d'A. senegal. En effet, la présence du gommier inhibe la croissance des cultures sous-jacentes. De nos jours, peu de données sont disponibles sur l'association de l'A. senegal avec les cultures en général et le niébé en particulier.

C'est donc pour contribuer à la promotion de l' Agroforesterie, à travers l'amélioration de la compréhension de l'interaction A.senegalcultures, que cette étude intitulée «Influence de l'Acacia senegal sur le sol et la production du niébé (Vigna unguiculata) à la station forestière de N'Dounga » a été initiée.

L'objectif principal assigné à ce travail est de connaître l'influence de 1'A. senegal sur la productivité du niébé. Il s'agit de manière spécifique d'évaluer l'effet fertilisant de l'A. senegal; de quantifier la production du niébé en interaction avec l'A. senegal et d'évaluer d'autres effets connexes comme le potentiel de fixation d'azote.

\section{Matériel et Méthodes}

\subsection{Matériel}

\subsubsection{Site expérimental}

L'essai a été réalisé à la station forestière de N'Dounga, une commune situé à $15 \mathrm{~km}$ de Niamey dans le département de Kollo, entre $2^{\circ} 18^{\prime} 28^{\prime}$ ' de longitude Est et $13^{\circ} 25^{\prime} 00^{\prime}$ ' de latitude Nord.

Le climat dans cette zone est de type sahélien; le régime pluviométrique est caractérisé par des précipitations irrégulières et mal réparties dans le temps et dans l'espace. Le maximum de volume se trouve concentré sur trois (3) mois (mi-juin à mi-septembre), étant le mois d'Aout le plus humide généralement. La pluviométrie moyenne annuelle est de $500 \mathrm{~mm}$ par an et la température maximale moyenne est de $36,9^{\circ} \mathrm{C}$ et la minimale de $22,7^{\circ} \mathrm{C}$. Le vent dominant est $1^{\prime}$ ' harmattan qui souffle pendant la saison sèche 
et la mousson pendant la saison des pluies (Rabiou et al., 2010). Durant la campagne 2015 la hauteur pluviométrique enregistrée était de 567,8 $\mathrm{mm}$ en 48 jours, une hauteur légèrement supérieure à la moyenne, le mois de juillet étant le plus pluvieux.

Le couvert végétal à la station est ouvert. Il est constitué de plantation artificielle composée de plusieurs espèces d'arbres et arbustes introduites la plupart dans le cadre des recherches effectuées à la station, pour les strates arborées et arbustives. Dans la station, la régénération naturelle des espèces locales est composée principalement par les Combrétacées (Guiera senegalensis, Combretum micranthum, Combretum glutinosum). Le tapis herbacé est tout aussi diversifié constitué de Cenchrus biflorus, Fagonia bruguieri, Pergularia tomentosa, Commelina benghalensis, Fimbristylis hispidula etc....

Le sol est de type ferrugineux tropicaux (classification Française et FAO), à texture sableuse à limono-sableuse, ayant une bonne profondeur (voir les autres caractéristiques dans la partie résultat analyse des sols).

\subsubsection{Matériel végétal}

Le matériel végétal utilisé dans le cadre de cette expérimentation est constitué des pieds groupés et isolés d'Acacia senegal mis en place lors d'un essai appelé « test de provenance d'Acacia local » en juillet 1987(un essai de provenances d'Acacia locaux et exotiques pour évaluation de la production de la gomme avec des fonds du projet IDA), et d'une variété de niébé précoce et érigée IT97K499-35 (variété d'IITA, Kano, Nigeria).

Pour les arbres isolés, trois (3) classes de diamètre du houppier des arbres ont été distinguées après la phase d'inventaire:] 2 ; 3],] 3 ; 4],] 4 ; 5] m. Par classe de diamètre du houppier, 4 arbres ont abrité les parcelles expérimentales. Les arbres qui sont choisis sont ceux dont le houppier n'interagit pas avec d'autres arbres.

\subsection{Méthodes}

La Figure 1 donne une schématisation non exhaustive des types d’opérations réalisées. 


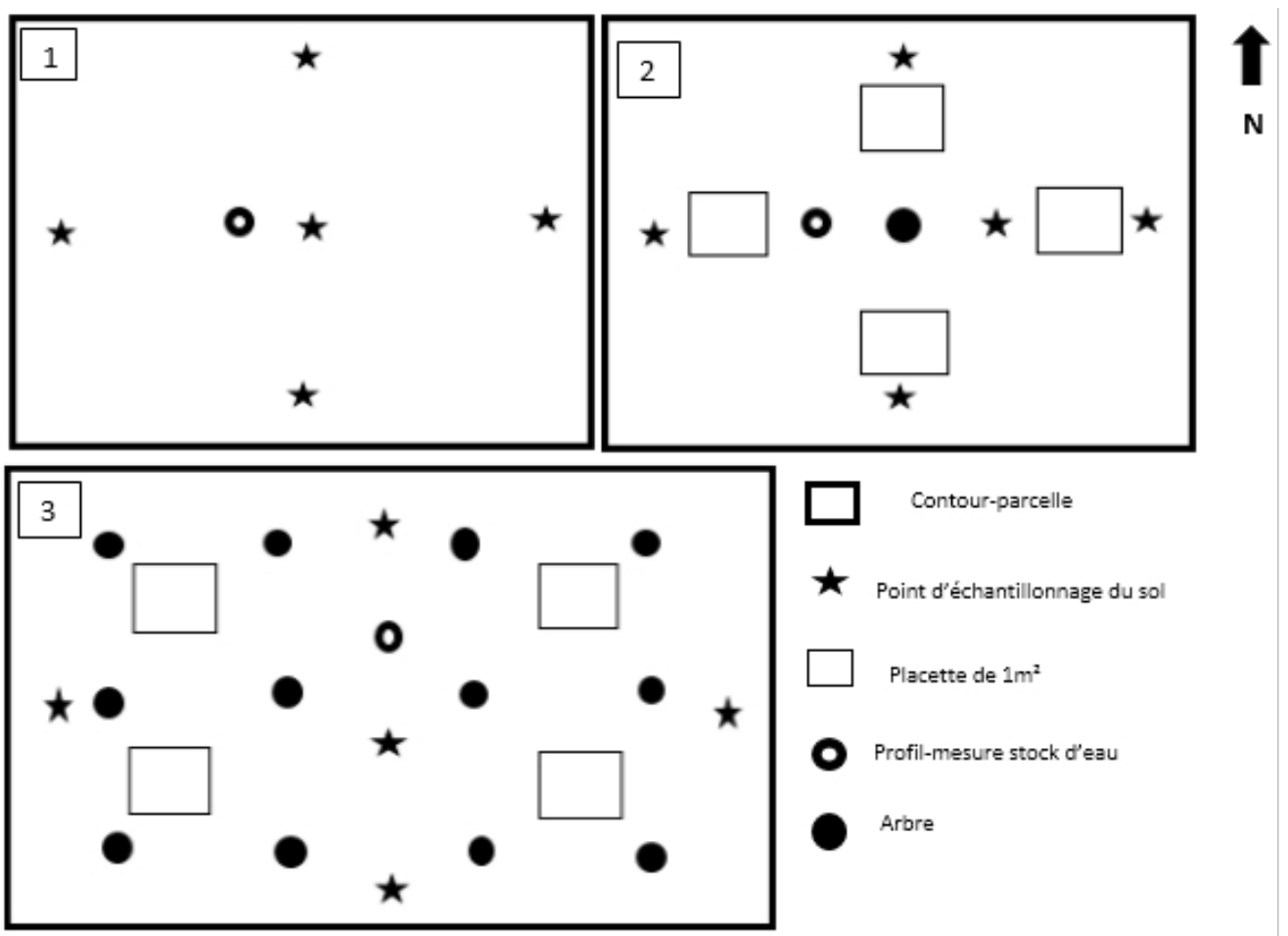

Figure 1. Graphique 1et 2 représentent un Acacia senegal isolé et le 3 plusieurs A. senegal en plantation.

\subsubsection{Echantillonnage du sol}

Avant la mise en place des cultures, un échantillonnage du sol du site expérimental a été réalisé. Les échantillons du sol ont été prélevés à la tarière à une profondeur de 0 à $20 \mathrm{~cm}$. Pour les parcelles témoins et les parcelles d'arbres groupés, cinq (5) échantillons ont été prélevés au milieu et au voisinage des quatre (4) angles. Ces échantillons ont ensuite été mélangés pour constituer un échantillon composite représentatif. Sous les houppiers d'arbres isolés, cinq (5) échantillons ont également été prélevés : un (1) au centre du rayon des houppiers et le reste dans les quatre (4) directions cardinales. Ces échantillons ont à leur tour été mélangés pour constituer un échantillon composite représentatif.

\subsubsection{Analyses chimiques du sol}

\section{Le pH}

La mesure du pH d'un sol permet de définir son statut acido-basique. Le $\mathrm{pH}$ joue un rôle prépondérant dans la vie des organismes du sol et dans la nutrition minérale des plantes. Il est définit par rapport aux ions $\mathrm{H}^{+}$et mesuré à l'aide d'un $\mathrm{pH}$-mètre de type HANNA minus d'une électrode en verre contenant une solution saturée de $\mathrm{KCl}$ (Mc Lean, 1982). 


\section{Azote total}

La détermination de l'azote total a été faite selon la méthode Kjeldahl (Bremmer \& Mulvaney, 1982).

\section{Carbone organique}

Le carbone a été dosé selon la méthode Walkley et Black (1934).

\section{Les bases échangeables et la capacité d'échange cationique}

$\mathrm{Ca}^{2+}, \mathrm{Mg}^{2+}, \mathrm{Na}^{+}$et $\mathrm{K}^{+}$, sont des cations liés au complexe absorbant. $\mathrm{La}$ détermination du $\mathrm{Na}^{+}$et $\mathrm{K}^{+}$a été réalisée au moyen du photomètre de flamme, ayant comme caractéristiques des sorties numériques et analogiques, détecteur de flamme et détecteur de pression d'air.

La détermination $\mathrm{du} \mathrm{Ca}^{2+}$ et $\mathrm{Mg}^{2+}$ a été faite par le spectrophotomètre à absorption atomique.

\section{Phosphore assimilable}

La méthode Bray 1 a été utilisée pour déterminer le $\mathrm{P}$ assimilable (Olsen \& Somers, 1982).

\section{Mise en place et suivi de la culture}

La méthodologie avait consisté à conduire en milieu réel un essai de culture du niébé (écart 0,70 x 0,20 m) sous houppier d'arbre isolé et sous frondaison fermée d'arbres (plantation) pour discerner l'effet « arbre », l'effet de « parc » et comparer au témoin sans arbre.

\section{Dispositif expérimental}

Le dispositif expérimental mis en place est un block complet randomisé éclaté à 4 répétitions. Chaque répétition comprend 5 parcelles correspondantes au nombre de traitements. Ces traitements se présentent comme suit :

$\checkmark$ Traitement 1 : «arbre isolé de]2;3] m de rayon de houppier »;

$\checkmark$ Traitement 2 : «arbre isolé de]3;4] m de rayon de houppier »;

$\checkmark$ Traitement 3 : «arbre isolé de]4; 5] m de rayon de houppier »;

$\checkmark$ Traitement 4 : « arbres groupés, plantation ou fourré d'arbre », (trois rangés d'arbres, espacés les uns des autres de $4 \mathrm{~m}$ ) ;

$\checkmark$ Traitement 5 : « témoin, sans arbre ».

\section{Préparation du terrain et semis}

Les travaux de préparation du terrain ont consisté à un labour superficiel à la hilaire.

Le semis a été effectué manuellement dans des poquets faits à la daba. Les écarts entre les lignes et entre les poquets étaient de 0,70 x 0,20 cm. Avant 
semis, les graines ont été traitées au fongicide contre l'éventuel germe qui causerait une fonte. Un démariage à deux (2) plants par poquet a été réalisé deux semaines après.

\section{Echantillonnage des plants et collecte des données}

Pour les différentes mensurations effectuées au cours de cette expérimentation, des carrés de rendements de $1 \mathrm{~m}^{2}$ ont été placés :

$\checkmark$ Arbre isolé : quatre (4) carrés dans les directions cardinales pour tenir compte du mouvement apparent du soleil, donc de l'ombrage ;

$\checkmark$ Arbres groupés : quatre (4) carrés étaient placés aléatoirement.

Enfin pour les témoins les parcelles entières ont été considérées $(2 \mathrm{~m} \times 2 \mathrm{~m})$.

Les variables mesurées concernaient :

\section{Nombre de nodosités}

Dix (10) plants étaient prélevés au moment de la floraison tout autour des carrés placés du fait du caractère destructif de cette observation. Les plants ont été récoltés manuellement et avec précaution à l'aide d'une pelle bêche enfoncée profondément et remuée autour du pied pour obtenir la plupart des parties basales des racines où se trouvent en général la majorité des nodules. La terre attachée aux racines a été délicatement éliminée à l'eau. Les plants sont par la suite séparés en parties aériennes et les parties racinaires portant les nodosités ont été prélevées et rincées sur place puis emballées dans des sacs plastiques pour permettre le comptage ultérieur au laboratoire (Peoples et al., 1989)

Les nodosités comptées ont été sectionnées transversalement dans le but d'observer la présence d'une coloration rouge- rose, verte ou blanche indicatrice $\mathrm{du}$ fonctionnement. Les colorations rouges et vertes sont indicatrices de la fixation d'azote. Plus particulièrement, la coloration rouge ou rosâtre indique la présence de la leghémoglobine qui transporte l'azote atmosphérique des racines jusqu'aux parties aériennes des plantes (Peoples et al., 1989).

\section{Quantification de la production}

Après la récolte, les gousses et les fanes ont été séchées durant une semaine au soleil ; ensuite les opérations suivantes ont été effectuées : Poids des gousses : il a été déterminé à l'aide d'une balance électronique ; Poids des graines : cette opération a consisté à décortiquer les gousses et les graines obtenues pesées à la balance électronique ;

Poids des fanes : les fanes ont aussi été pesées après leur séchage à l'aide de la balance électronique ;

Ces différents poids mesurés ont servi au calcul de différents rendements. 


\section{Paramètres calculés}

Pour les trois premiers paramètres mesurés, les rendements correspondant ont été calculés.

Pour pouvoir apprécier l'effet d'A. senegal sur la production du niébé, les gousses, les graines et les fanes à la récolte ont été pesées à la balance, puis les rendements (en $\mathrm{Q} / \mathrm{ha}$ ) correspondant ont été calculés.

\subsubsection{Traitements des données}

Les données collectées ont été traitées au logiciel Minitab 16 et les moyennes des variables ont été comparées en utilisant le test de Turkey au seuil de probabilité $\mathrm{p}=5 \%$ dans le cas où il y a un effet significatif $(\mathrm{p}<5 \%)$.

\section{Résultats et Discussion}

\subsection{Effet d'A. senegal sur le sol}

Variation des paramètres chimiques du sol sous et hors houppiers

Le Tableau 1 donne les résultats d'analyse de variance des différents paramètres chimiques du sol sous et hors houppier.

Tableau 1. Analyse de la variance des éléments et paramètres chimiques des sols

\begin{tabular}{|c|c|c|c|c|c|c|c|}
\hline Traitements & $\mathrm{pH} \mathrm{H} 2 \mathrm{O} \mathrm{i}$ & $\mathrm{pH} \mathrm{KCli}$ & $\mathrm{Ca}^{2}+\mathrm{i}$ & $\mathrm{Mg}^{2}+\mathrm{i}$ & $\mathrm{Na}+\mathrm{i}$ & $\mathrm{k}+\mathrm{i}$ & $\mathrm{Si}$ \\
\hline Arbre 1 & $5,91 \mathrm{a}$ & 5,09 a & $42375 \mathrm{~b}$ & $1,0675 \mathrm{a}$ & $0,145 \mathrm{a}$ & $0,1425 \mathrm{a}$ & $5,592 \mathrm{~b}$ \\
\hline Arbre 2 & $5,51 \mathrm{a}$ & $4,7175 \mathrm{a}$ & $4,0875 \mathrm{~b}$ & 0,9375 a & $0,1425 \mathrm{a}$ & $0,1075 \mathrm{a}$ & $5,275 \mathrm{~b}$ \\
\hline Arbre 3 & $5,3175 \mathrm{a}$ & $4,1625 \mathrm{a}$ & $4,8125 \mathrm{ab}$ & $1,0075 \mathrm{a}$ & $0,115 \mathrm{a}$ & $0,075 \mathrm{a}$ & $6,01 \mathrm{ab}$ \\
\hline control & $3,84 \mathrm{~b}$ & 3,79 a & $5,55 \mathrm{ab}$ & $0,81 \mathrm{a}$ & 0,18 a & $0,07 \mathrm{a}$ & $6,61 \mathrm{ab}$ \\
\hline plantation & $5,6725 \mathrm{a}$ & $5,1825 \mathrm{a}$ & $6,675 \mathrm{a}$ & $1,0925 \mathrm{a}$ & $0,1575 \mathrm{a}$ & $0,1125 \mathrm{a}$ & $8,037 \mathrm{a}$ \\
\hline probabilité $(5 \%)$ & 0,000 & 0,032 & 0,007 & 0,384 & 0,088 & 0,123 & 0,023 \\
\hline Traitements & $\mathrm{CEC}_{\mathrm{i}}$ & $\mathrm{P}$ ass $\mathrm{i}$ & P total i & C. ogr $(\%) \mathrm{i}$ & M.o (\%)i & $\mathrm{N}(\%) \mathrm{i}$ & $\mathrm{C} / \mathrm{Ni}$ \\
\hline Arbre 1 & $5,048 \mathrm{~b}$ & $6,072 \mathrm{a}$ & $1023,9 \mathrm{a}$ & $0,815 \mathrm{a}$ & 0,5425 a & $0,1305 \mathrm{a}$ & $6,191 \mathrm{a}$ \\
\hline Arbre 2 & $5,565 \mathrm{ab}$ & $5,362 \mathrm{a}$ & $1020,4 \mathrm{a}$ & 0,2675 a & $0,46 \mathrm{a}$ & $0,1305 \mathrm{a}$ & $2,044 \mathrm{a}$ \\
\hline Arbre 3 & $6,24 \mathrm{~b}$ & $4,961 \mathrm{a}$ & $780,6 \mathrm{a}$ & $0,2425 \mathrm{a}$ & $0,4125 \mathrm{ab}$ & $0,12425 \mathrm{a}$ & $1,955 \mathrm{a}$ \\
\hline control & $7,12 \mathrm{ab}$ & $1,89 \mathrm{a}$ & $499,5 \mathrm{a}$ & $0,11 \mathrm{a}$ & $0,19 \mathrm{~b}$ & $0,112 \mathrm{a}$ & $0,982 \mathrm{a}$ \\
\hline plantation & $8,13 \mathrm{a}$ & $13,752 \mathrm{a}$ & $980,1 \mathrm{a}$ & $0,29 \mathrm{a}$ & $0,4975 \mathrm{a}$ & $0,12225 \mathrm{a}$ & $2,342 \mathrm{a}$ \\
\hline probabilité $(5 \%)$ & 0,008 & 0,138 & 0,316 & 0,253 & 0,005 & 0236 & 0,267 \\
\hline
\end{tabular}

Les moyennes affectées par la même lettre sur la même colonne ne sont pas statistiquement différentes au seuil de 5\% par le test de Tukey.

\section{a. Le potentiel d'hydrogène $(\mathrm{pH})$}

Il ressort de l'analyse que le $\mathrm{pH}$ est acide quelle que soit la modalité (Tableau 1). Cependant, cette acidité diminue en présence d' A. senegal (5,91 au niveau de l'Arbre 1 contre 3,54 pour le témoin). Cette régression se fait également suivant la grandeur de la classe du houppier, grandeurs qui se trouvent toutes être supérieures à celle du témoin (sans arbre). Il en est de 
même pour le $\mathrm{pH} \mathrm{KCl}$. L'analyse de la variance montre une différence hautement significative entre le contrôle et les autres traitements pour le $\mathrm{pH}$ eau $(\mathrm{P}<0.0001)$. Ces résultats ne sont pas conformes à ceux obtenus par (Samb, 2009; Abdou et al., 2014) qui avaient trouvé une différence non significative entre les valeurs du pH sous et hors houppier. Cette augmentation du $\mathrm{pH}$ dans les sols sous houppier d'arbre isolé ou groupés peut s'expliquer par les effets de la décomposition par les microorganismes du sol de la litière formée par les feuilles des Acacias et les produits de leur exsudation racinaire. La décomposition de cette litière dans le sol produit de nombreux cations $\left(\mathrm{Ca}^{+}, \mathrm{Mg}^{+}, \mathrm{K}^{+}\right.$, etc.) qui neutralisent l'acidité du sol et augmentent le $\mathrm{pH}$.

\section{b. Bases échangeables}

Les valeurs les plus élevées sont enregistrées au niveau de la Plantation (8.13) et du control (7.12) suivi des arbres 3 et 1 (Tableau 1). Par contre, la plus faible se retrouve au niveau d'Arbre 2. Cet état de fait est présidé par l'ion $\mathrm{Ca}^{2+}$ lequel présente des valeurs élevées par rapport aux autres composantes de la somme des bases qui néanmoins sont plus élevées majoritairement en présence d'A. senegal. L'analyse de la variance présentée au Tableau 1 montre qu'il n'existe pas de différence significative au sein de chacune des modalités dans la teneur en ses éléments $\left(\mathrm{Mg}^{2}+, \mathrm{Na}^{+}\right.$et $\left.\mathrm{K}^{+}\right)$. Par contre, pour la teneur en $\mathrm{Ca}^{2}+$ il existe une différence significative $(\mathrm{P}<0.007)$ entre les traitements Plantation et Arbre 1, Arbre 2. Ce même constat est fait au niveau global, en l'occurrence au niveau du système international, Si. Les valeurs élevées constatées de $\mathrm{Ca}^{2}+$ peuvent être expliquées par le fait que la fixation des cations sur le complexe suit un ordre préférentiel, le cation $\mathrm{Ca}^{2}+\mathrm{se}$ trouvant dans les premiers rangs de cet ordre selon le type d'argile présent. Cela peut être possible aussi par sa relative abondance dans les sols du site par rapport aux autres éléments.

\section{c. Capacité d'échange cationique (CEC)}

La CEC varie entre 5.04 et 8.13 méq/100g pour tous les traitements confondus (Tableau 1). La plus grande valeur a été enregistrée au niveau de la Plantation suivie du contrôle, et pour les arbres isolés la valeur du CEC augmentait au fur et à mesure que la classe de l'arbre diminue. Ces valeurs sont basses (Agro Consult, 1987) mais elles sont supérieures au seuil critique de 4 méq/100g de sol. Cette baisse généralisée peut être due à des valeurs faibles du pH selon Anonyme (2013). L'augmentation des valeurs faibles du $\mathrm{pH}$ se traduit généralement par des colloïdes négatifs apparus par une augmentation des charges négatives. Inversement, l'acidification du sol se traduit par la diminution de l'adsorption cationique, et sa hausse au niveau de certain par rapport à la matière organique. L'augmentation de la valeur du 
CEC constatée au niveau du contrôle pourrait être liée au passé cultural du site abritant la parcelle correspondante à ce traitement.

\section{d. Phosphore assimilable}

Le phosphore assimilable varie de 1.89ppm (control) à 13,75ppm dans les plantations (Tableau 1). Ces résultats sont similaires à ceux obtenus par El Atta et al. (2010) sur plusieurs espèces d'Acacia et Bazongo et al. (2015) et sur d'autres espèces comme Detarium microcarpa et Jatropha. curcas. La teneur élevée en phosphore assimilable constatée sous Plantation quant à elle pourrait être liée à la symbiose racinaire avec les champignons mycorhiziens (Communication personnelle). Mais aussi selon Kaci et al. (2002) la teneur du sol en phosphore assimilable augmente à proximité des racines par rapport au sol distal, ce qui se traduit par la libération, de la part des racines, d'ions $\mathrm{H}+$ qui dissolvent les phosphates naturels en les rendant assimilables.

\section{e. Matière organique}

Les teneurs en matière organique et en carbone organique sont plus élevées au niveau du traitement Arbre 1 suivit du traitement plantation, et les autres se suivent selon l'ordre décroissant du rayon du houppier, mais elles sont toutes supérieures aux teneurs trouvées au niveau du témoin $(0.19 \%)$. Toutefois l'analyse de la variance montre qu'il n'y a pas de différence significative entre les traitements. Des résultats similaires ont été trouvés sous l'espèce A. senegal (El Tahir et al., 2004).

Les teneurs observées au niveau du traitement Arbre 1 peuvent être expliquées par une erreur de prise d'échantillon du sol. En effet, la prise aurait peut-être coïncidé avec un substrat en décomposition. Par contre, les autres teneurs sur le reste des traitements peuvent être proportionnelles aux apports faits par les arbres en litière $(0.40 \%)$.

\section{Azote}

La teneur en azote dans les sols des différents traitements est plus élevée sous houppier (arbres isolés et plantation) par rapport au témoin, mais le résultat de l'analyse de la variance n'a révélé aucune différence significative. La teneur plus élevée en azote sous houppier d'arbre et en plantation pourrait être attribuée à la fixation biologique de l'azote, à la décomposition des résidus d'arbres et à la poussière atmosphérique emprisonnée par l'arbre (E1 Tahir et al., 2009). Ces teneurs se rapprochent à celles trouvées par Samb (2010), mais corroborent les résultats des deux études. 


\section{Rapport C/N}

Le rapport $\mathrm{C} / \mathrm{N}$ est plus élevé au niveau du traitement Arbre 1 suivi de la plantation et des autres traitements et il est moins important au niveau des témoins. Cette dynamique est la même que celle de la matière organique. Ces valeurs enregistrées prouvent de fort taux de minéralisation de la matière organique sous les différents traitements; cependant l'analyse de la variance n'a révélée aucune différence significative. Ces résultats sont en désaccord avec ceux obtenus par Githae et al. (2001) qui a trouvé un ratio plus élevé hors houppier d'A.senegal que sous son houppier, donc une minéralisation plus poussée sous houppier. Le $\mathrm{C} / \mathrm{N}$ plus élevé au niveau du témoin peut être expliqué par le faible apport de la matière organique par rapport aux autres traitements.

\subsection{Potentiel de fixation d'azote}

De manière générale sur tous les traitements, les nombres de nodules et nodosités efficaces enregistrés étaient faibles. La comparaison des moyennes des différents traitements effectués, n'a révélé aucune différence significative $(\mathrm{P}>0.05)$.

Ces faibles nodosités pourraient être amputables non seulement à la méthode de prélèvement mais aussi à l'acidité du sol. En effet, sous houppier il peut y avoir une imbrication des racines du niébé due à la forte concentration des racines de niébé ou des arbres pouvant induire la cassure des racines du niébé lors des prélèvements et donc la perte de ses nodules. L'acidité du sol des parcelles est aussi une des causes, car selon Cacciari et al. (2000) une interaction entre le $\mathrm{pH}$ et des températures élevées peut avoir des effets plus néfastes sur la symbiose que chacun de ces facteurs pris séparément. Il convient cependant de noter des nombres plus élevés en nodosités et nodosités efficaces ont été observés au niveau des arbres isolés, respectivement arbre 1, 2 et 3 , et également au niveau de la plantation, donc des nombres plus élevés sont observés sous houppier par rapport au témoin. Ceci s'explique par le niveau d'acidité moins élevé sur ces sols par rapport au contrôle, mais également ceci peut être lié aux températures moins élevées dues à l'ombrage occasionné par la frondaison des arbres. Par ailleurs, Samb (2010) a noté la présence massive de population microbienne sous houppier d'A. senegal, raison pour laquelle il y a une possibilité plus grande d'existence de diverses souches de rhizobia capables d'association symbiotique. 


\section{Nombre, nodosités totales et nodosités eficaces}

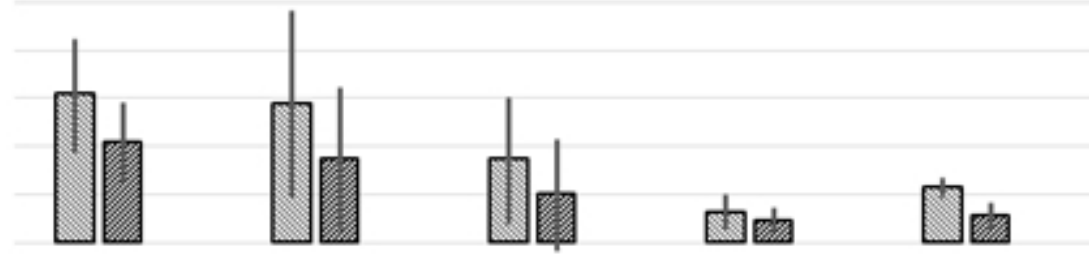

$\begin{array}{llll}\text { Arbre } 1 & \text { Arbre } 2 & \text { Arbre } 3 & \text { Control }\end{array}$

Plantation

\section{صnodosités totales $\boldsymbol{\square}$ nodosités efficaces}

Figure 2. Quantité de nodosités par unité d'expérimentation

\section{3. $\quad$ Effet d'A.senegal sur la production}

Il ressort de cette étude que le témoin a les meilleures performances sur tous les paramètres mesurés pour la production, suivi du traitement Arbre 2 et ensuite du traitement Arbre 1, puis de la Plantation et en fin le traitement Arbre 3 qui a été le moins performant (Tableau 2). Le résultat de la comparaison des moyennes des traitements ci-dessus présentée montre des différences hautement significatives $(\mathrm{P}<0.0001)$.

Pour le rendement en fane le témoin se distingue complètement des autres traitements (Figure 3). Par contre, les autres différences observées étaient hautement significatives mais présentent des valeurs intermédiaires.

Ces résultats dénotent globalement l'effet dépréciatif d' A. senegal sur la production des cultures sous-jacentes et infirment notre hypothèse, confirmant du coup les dires des paysans rapportés par PAC II en 2011. Des résultats similaires ont été observés par Mansour et al. (2016) dans la zone sous influence du houppier et proche du tronc, cas d'associations A. senegal avec le sorgho et avec le mil. Pourtant, ils ne corroborent ceux obtenues par Abdou (2006), qui avait trouvé une hausse en production de biomasse du mil en fonction de la grandeur décroissante du houppier. Selon Sabir et Roose (2010), la plantation d'arbres forestiers inhibe la croissance des herbes fourragères exploitées par le bétail. Des améliorations du statu physicochimique du sol sous houppier ont été observées, notamment le $\mathrm{pH}$ qui conditionne l'absorption des éléments minéraux par les plantes au niveau du stock d'eau du sol, bien que le model ait été insuffisant pour une analyse de la variance des améliorations qui étaient observées au niveau du traitement 
Plantation pendant le «séjour» des plantes de niébé. Au vue de l'amélioration de tous ces facteurs au niveau des sols sous influence du houppier d'A. senegal par rapport au témoin, la diminution de ces rendements laisse présager un effet dépressif de cette espèce sur les cultures.

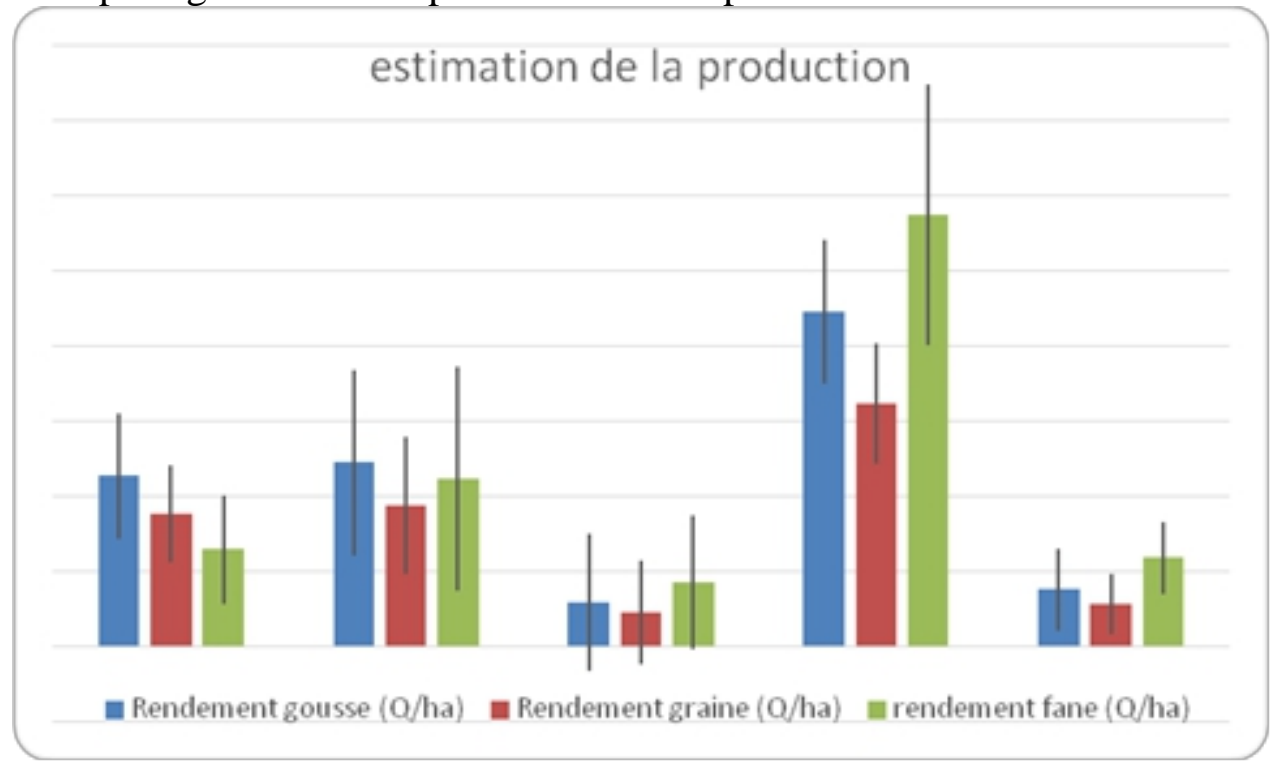

Figure 3. Rendements fanes, gousses, graines suivants les traitements

Tableau 2. Analyse de la variance des différents rendements

\begin{tabular}{|c|c|c|c|}
\hline Traitements & Rendement gousse ( $\mathrm{Q} / \mathrm{ha})$ & Rendement graine $(\mathrm{Q} / \mathrm{ha})$ & rendement fane $(\mathrm{Q} / \mathrm{ha})$ \\
\hline Arbre 1 & $4,53 \mathrm{~b}$ & $3,53 \mathrm{ab}$ & $2,57 \mathrm{~b}$ \\
\hline Arbre 2 & $4,90 \mathrm{ab}$ & $3,74 \mathrm{ab}$ & $4,45 \mathrm{~b}$ \\
\hline Arbre 3 & $1,16 \mathrm{~b}$ & $0,91 \mathrm{~b}$ & $1,71 \mathrm{~b}$ \\
\hline témoin & 8,91 a & $6,47 \mathrm{a}$ & 11,48 a \\
\hline plantation & $1,50 \mathrm{~b}$ & $1,14 b$ & $2,35 \mathrm{~b}$ \\
\hline probablité (5! & 0,000 & 0,000 & 0,000 \\
\hline
\end{tabular}

Les moyennes affectées par la même lettre sur la même colonne ne sont pas statistiquement différentes au seuil de $5 \%$ par le test de Tukey.

\subsection{Dynamique des paramètres chimiques}

Il est à noter des hausses de paramètres chimiques au niveau des arbres isolés par rapport au témoin et à la Plantation où des régressions par rapport à l'état initial étaient constatées (Tableau 3). Il est également constaté une accentuation de l'acidification du sol sur les traitements en présence d'A. senegal.

Les augmentations peuvent être dues à une restitution faite par la litière produite par les deux espèces en présence aussi du contenu de la forte minéralisation de la matière organique. Au niveau de la Plantation l'humidité 
régnante avait entretenu les plantes de niébé sans sénescence des feuillages jusqu'à la récolte, donc un apport moindre par rapport aux traitements, ceci explique également la réduction de l'azote constaté qui serait peut être utilisé par les micro-organismes pour cette minéralisation (Zanguina et al., 2018).

Tableau 3. Paramètres chimiques en fin d'essais

\begin{tabular}{|c|c|c|c|c|c|c|c|}
\hline Traitements & $\mathrm{pH} \mathrm{H} 2 \mathrm{O}$ & $\mathrm{pH} \mathrm{KCl}$ & $\mathrm{Ca}+$ & $\mathrm{Mg}++$ & $\mathrm{Na}+$ & $\mathrm{k}+$ & $\mathrm{S}$ \\
\hline Arbre 1 & $-0,1425$ & 0,175 & 1,8625 & 2,4775 & 0,03 & 0,007 & 4378 \\
\hline Arbre 2 & $-0,295$ & 0,12 & 0,7875 & 1,7675 & 0,0275 & 0,0325 & 2,615 \\
\hline Arbre 3 & $-0,5025$ & 0,2075 & 0,7875 & 1,53 & 0,04 & 0,03 & 2388 \\
\hline Témoin & 0,82 & 0,09 & $-2,7$ & 0,33 & $-0,06$ & $-0,01$ & $-2,44$ \\
\hline plantation & $-0,405$ & $-0,2025$ & $-2,85$ & 1,1975 & $-0,0075$ & 0,0225 & $-1,637$ \\
\hline Traitements & $\mathrm{CEC}$ & $\mathrm{P}$ ass & P total & C. ogr & M.o (\%) & $\mathrm{N}(\%)$ & $\mathrm{C} / \mathrm{N}$ \\
\hline Arbre 1 & 4,98 & 0,94 & 95,4 & $-0,195$ & 0,5225 & $-0,003$ & $-1,3057$ \\
\hline Arbre 2 & 2,382 & 1,56 & 24,3 & 0,065 & 0,1 & $-0,01875$ & 0,9307 \\
\hline Arbre 3 & 2,313 & $-0,646$ & 386,7 & 0,0375 & 0,0725 & $-0,0155$ & 0,6383 \\
\hline Témoin & $-2,45$ & 0,18 & 285,9 & 0,07 & 0,13 & 0,0145 & 1,9312 \\
\hline plantation & $-0,417$ & 0,206 & $-4,8$ & 0,075 & 0,1275 & 0,00425 & $-0,1733$ \\
\hline
\end{tabular}

\section{Conclusion}

Il ressort de cette étude que la présence de l'espèce $A$. senegal est d'une grande importance car elle crée un environnement agro-écologique favorable, notamment à l'amélioration de la fertilité des sols, comme l'avaient mis en évidence plusieurs auteurs auparavant. En effet, les mesures quantitatives et qualitatives de certains paramètres de fertilité des sols ont permis de constater que le sol se trouvant sous l'influence du houppier de l'arbre est riche en éléments minéraux. Outre ces facteurs il a également été mis en évidence un potentiel plus élevé de capacité de symbiose rhisobienne. Malgré ces améliorations, il existe encore un facteur qui empêche l'expression du potentiel en rendement du niébé : la zone se trouvant sous houppier d'A. senegal. Cependant, la dynamique constatée des éléments minéraux après récoltes rassure les paysans sur la durabilité du système (système arbre/culture).

\section{References:}

1. Abdou, M. M., Zoubeirou, A. M., Dan Lamso, N., \& Ambouta, J. M. K. (2014). Productivité de la culture du sorgho (Sorghum bicolor) dans un système agroforestier à base d'Acacia senegal (L.) Willd. Au Niger. Journal of Applied Biosciences 82:7339-7346.

2. Bado, B.V. (2002). Rôle des légumineuses sur la fertilité des sols ferrugineux tropicaux des zones Guinéenne et Soudanienne du Burkina Faso. Thèse de Doctorat : Université de Laval (Québec). 184 p. 
3. Bazongo, P., Traore, K., Traore, O., Yelemou, B., Sanon, K.B., Kabore, S.,Victor Hien, V., \& Nacro, B.H. (2015). Influence des haies de Jatropha sur le rendement d'une culture de sorgho (Sorghum vulgare) dans la zone Ouest du Burkina Faso: cas du terroir de Torokoro .Int. J. Biol. Chem. Sci. 9(6): 2595-2607.

4. Boubacar, A. (2006). Effet du niveau d'élagage et de la distance du pied d'Acacia senegal (L.) Willd sur la production du mil Pennisetum glaucum (L.) R.Br. en station. Mémoire d'Ingénieur des Techniques Agricoles, Faculté d'Agronomie, Université Abdou Moumouni de Niamey, 41P.

5. Bremner, JM. \& Mulavaney, CS. (1982). "Nitrogen total," in American Society of Agronomy, Madison, Wis, USA.

6. Cacciari, I. D. I., Mattia, E., Quatrini, R., Catelli, M. C., Grego, M. O. S., Lippi, D., M., \& Paolis, R. D.E. (2000). Réponses adaptatives des isolats de Rhizobium aux stress. Soil Biol. Biochem., 17 : 797-802.

7. El Atta, H., Aref, I., \& Ahmed, A. (2013). Effect of Acacia spp. on soil properties in the highlands of Saudi Arabia. Life Science Journal; 10 (4).

8. El Tahir, B.A., Ahmedb, D.M., Ardo, J., Gaafar, A.M., \& Salih, A.A. (2009). Changes in soil properties following conversion of Acacia senegal plantation to other land management systems in North Kordofan State, Sudan. Journal of Arid Environments 73 (2009) 499505. 51 .

9. El Tahir, B.A., Madibo, G.M., \& El Wakeel, A.S. (2004). Influence of Acacia senegal, Acacia seyal and Acacia tortilis on some properties of sandy soil in North Kordofan State, Sudan. University of Khartoum Journal of Agricultural Sciences, 12(1), 127-141.

10. Gbemavo, D.S.J.C., Glèlè Kakaï, R., Assogbadjo, A.E., Katary, A., \& Gnanglè, P. (2010). Effet de l'ombrage du karité sur le rendement capsulaire du coton dans les agroécosystèmes coton-karité du Nord Bénin. Tropicultura. 28 (4): 193-199.

11. Githae, E.W., Gachene, C.K., Jesse, T., \& Njoka, J. T. (2011). Soil physicochemical properties under Acacia senegal varieties in the dryland areas of Kenya. Afr. J. Plant Sci.5 (8), pp. 475-482.

12. Grouzis, M. \& Akpo, L.E. (2006). Interactions arbre-herbe au Sahel. Sécheresse 17 (1-2) : 318-25.

13. Ibrahim, M.B. (2010). Contribution à l'évaluation de la diversité de la durée des cycles de variétés précoces et tardives de mil issues de quatre localités du Niger. Mémoire de Master II, Faculté d'Agronomie, Université Abdou Moumouni de Niamey, 41P. 52.

14. Kaci, M.B.N., Hedde, M., Mouas-Bourbia, S., Siad, D., \& Derridj, A. (2007). Biodisponibilité du phosphore dans la rhizosphère du 
grenadier (Punica granatum L.) Sous climat aride : cas des vergers de Messaad Wilaya de Djelfa.UMMTO Algérie.

15. Koulibaly, B., Traoré, O., Dakuo, D., Zombré, P.N., \& Bondé, D. (2010). Effets de la gestion des résidus de récolte sur les rendements et les bilans culturaux d'une rotation cotonnier-maïs-sorgho au Burkina Faso. Tropicultura, 28: 184-189.

16. Larwanou, M., Oumarou, I., Snook, L., Dan guimbo, I., \& Eyog-Matic, O. (2010). Pratiques sylvicoles et culturales dans les parcs agroforestiers suivant un gradient pluviométrique nord-sud dans la région de Maradi au Niger. Tropicultura, 28 (2) : 115-122.

17. Mclean, E.O. (1982). Soil pH and Lime Requirement. In Page et al (eds). Methods of Soil Analysis, part 2, Chemical and Microbia Properties 2nd 256 ed. Agronomy 257 Monograph No 9, ASA and SSSA, Madison, WI.

18. PACII (2011). Etude de cas pour la validation du standard CCB. Projet de plantation d'Acacia senegal. Unité de gestion et de suivi. 103P.

19. Rabiou, A., Toujani, A., Jensen, J. S., Raebild, A., \& Allassan, M. (2010). Rapport de mise en place d'un verger à graine à Baobab (Adansonia digitata L.) à la station experimental de N'Dounga, Niger Landscape Working Paper no. 49-2010.

20. Raddad, E.Y. \& Luukkanen, O. (2006). The influence of different Acacia senegal agroforestry systems on soil water and crop yields in clay soils of the Blue Nile region, Sudan. agricultural water management 87 ( 2007 ) $61-72$.

21. RECA (2010). Essai d'estimation des quantités d'engrais utilisées pour la campagne hivernale. Note d'information/ intrants $n^{\circ} 13$.

22. Olsen \& Sommers (1982). Phosphorus. In Page et al (eds), Methods of Soil Analysis, part 2, 2nd 261 ed. Agronomy Monograph No 9, ASA and SSSA, Madison, WI.

23. Sabir, M. \& Roose, E. (2010). Gestion durable des eaux et des sols au Maroc : Valorisation des techniques traditionnelles méditerranéennes IRD Ed-343p.

24. Samb N., 2010. Influence d'Acacia senegal (1.) willd sur les propriétés physiques, chimiques et biologiques des sols. Mémoires d'études approfondies (DEA) de biologie végétale. Université CHEIK ANTA DIOP de Dakar, 69 pages.

25. Walkley, A. \& Black, C.A. (1934). An examination of the degtjareff method for determining soil organic matter and a proposal modification of the chromic acid titration method. Soil Sci., 37 (1): 2938.

26. Zanguina A, Mella M. T, Addam K S, Ousmane G 2018. Development of Compost from Agro-food Residues: Analysis of the Crop Nutrients 
and Trace Elements. American Scientific Research Journal for Engineering, Technology, and Sciences (ASRJETS) (2018) Volume 43, No 1, pp 1-12.

27. Zomboudré, G., Zombré, G., Ouédraogo, M. B., Guinko, S., \& Macauley, H. R. (2005). Réponse physiologique et productivité des cultures dans un système agroforestier traditionnel : cas du maïs (Zea mays L.) associé au karité (Vitallaria paradoxa Gaertn) dans la zone Est du Burkina Faso. Biotechnol. Agron. Soc. Environ., 9 (1) : 75-85 56. 